Journal of Engineering and Applied Sciences 14 (3): 744-749, 2019

ISSN: 1816-949X

(C) Medwell Journals, 2019

\title{
Study on Dual-Mode Microstrip Slotted Patch Filter Using Perturbation Element
}

\author{
Riyadh Khlf Ahmed \\ Department of Communication Engineering, College of Engineering, \\ University of Diyala, Baqubah, Iraq
}

\begin{abstract}
In this research article, an investigation on dual-mode microstrip bandpass filter has been given. It discusses the effect of various dual-mode resonators used in the literature with diverse kinds of perturbation elements on frequency response of microstrip filter. A new compacted microstrip bandpass filter using new slotted dual-mode resonator has been simulated and analyzed. This filter hassimulated at $5.7 \mathrm{GHz}$ with return loss and insertion loss values of 1.69 and $10.31 \mathrm{~dB}$. Also, this designed filter has perceptibly narrow fractional bandwidth of $2.22 \%$ ( $127 \mathrm{MHz}$ from its center frequency). Consequently, the modeled microstrip filter can be practical and integrated within many communication schemes and devices.
\end{abstract}

$\underline{\text { Key words: Microstrip filter, slotted patch resonator, dual-mode principle, compactness, bandpass, frequency }}$

\section{INTRODUCTION}

Recently wireless communication systems demand highly a compact microstrip narrow Bandpass Filters $(\mathrm{BPFs})$, due to their ease of manufacturing and great discrimination of these filters. The Bandpass (BPF) design could have highly desirable features in terms of smallness, small radiation loss and design simplicity by using dual-mode resonators. Actually, the dual-mode microstrip filters are extremely imperative in smallness methods because of their extraordinary acquired performance and they are possibly employed as a paired circuit in single configuration unlike solitary mode bandpass filter designs (Mezaal et al., 2013; Mezaal and Eyyuboglu, 2016).

For years, the wireless communications systems need the miniature excellent performance microwave BPFs. Therefore, the dual-mode filters are employed extensively for these schemes due to their features which are compactness, low loss and weight besides high selectivity.

Numerous wideband BPFs have been realized by means of dual-mode ring resonators with tuning stubs but the topology of these filters still take a big space that is improper for wireless classifications where the smallness is an remarkable aspect. Accordingly, it is essential to create innovative forms of dual-mode microstrip resonators not merely for proposing different designs but likewise for diminishing filters.

The concept of the dual-mode stands for double degenerate modes of a structural regular resonator. On the other hand, each dual-mode resonator is possibly adopted as a twice tuned resonant circuit that leads to a compacted filter formation (Gupta, 2017).
This dual mode notion is distinct to the whole regular resonators which are capable to degenerate modes at similarmid-band frequency. If the symmetry is exist, the dualistic modes are orthogonal and they cannot switch the microwave power. In contrast, if the structural symmetry is appropriately cracked, the conditions of resonator boundary exchange permitting the coupling amid the modes. Therefore, dual modes are existing at marginally different frequencies.

These two degenerate modes can be induced instantaneously that co-exist autonomously of each other by adding the perturbation notches along the ring (Hong and Lancaster, 2001).

The perturbation elements can be a small cut to electromagnetically disturb the square patch and disk resonators or they can be small square patch to insert them into the ring, square loop meander loop resonators as in Fig. 1. However, other forms of perturbations may also use for coupling the dualistic degenerate modes. For example, a diminutive circular patch or disk can be used for pairing the double degenerate modes and in the similar manner, a rectangular patch is possibly used in place of square patch for the coupling (Hong and Lancaster, 1995a, b).

According to Babinet's principle, the slots can be depicted as resonant radiator. The French Physicist and Mathematician, Jacques Babinet (1794-1872) created the proposition that comparable diffraction patterns are formed by dual corresponding screens. The radiated field and impedance of an aperture or slot antenna are similar to this principle (Hong and $\mathrm{Li}, 2004$ ).

Planar technology has been still acting a great part in the subject of microwave circuits and systems (Wolff, 
(a)

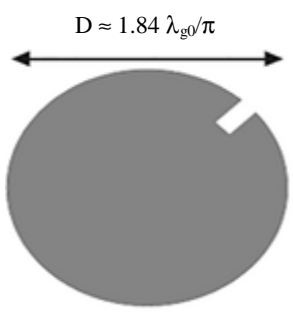

(c)

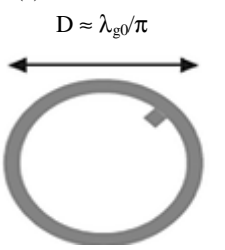

(b)

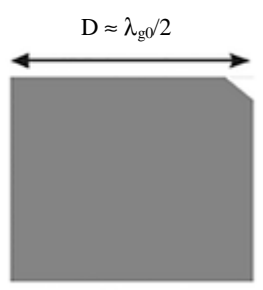

(d)

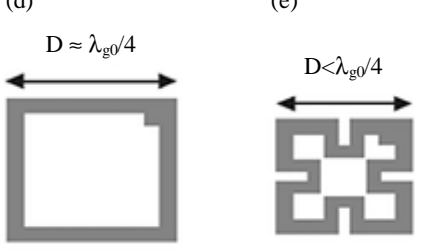

Fig. 1a-e): General used dual-mode resonators

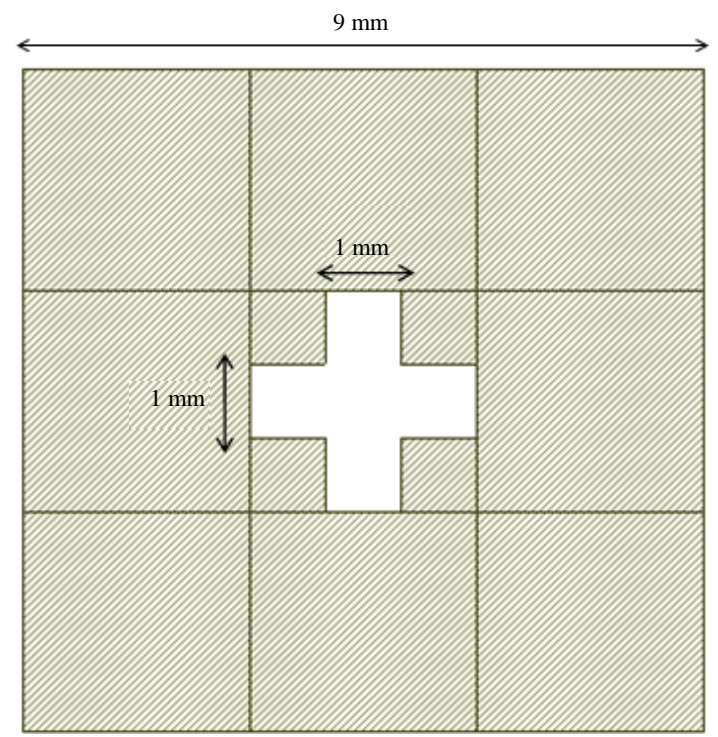

Fig. 2: The modeled slotted patch resonator

1972). The use of dual-mode in microstrip filter is primarily created by Wolf where 2 nd order is realized using new structure which requires only two earthed capacitors (Kundu and Awai, 2001).

Primarily, a dual-mode microstrip square loop resonator is investigated to design compact microwave bandpass filters where the features of mode splitting were designated (Hong and Lancaster, 1995a, b).

A degenerate mode of meander loop resonator was exploited to create new sort of dual-moazde microstrip $\mathrm{BPF}$ for compacting high selectivity narrowband microwave bandpass filters.
The dual-mode operating of microstrip triangular patch resonators and its uses for constructing dual-mode BPFs were investigated by Sheta (2008). Microstrip slow-wave open-loop resonator filters are presented with the theory and experiment (Sheta et al., 2006; Singhal et al., 2010). Introduce a new nondegenerate dual mode microstrip patch filter structure where the filter is developed on square microstrip patch with four slots etched in symmetrical style. A non-degenerate dual-mode resonator with open circuit stubs at dual opposite corners can be used to develop compact narrowband filter (Sun and Zhu, 2014; Yeo and Nwajana, 2013).

The mode perturbing components of a $2 \mathrm{D}$ ring resonator were studied for an application and integrated topology of wideband and multiband microwaves filters (Hong and Lancaster, 1995a, b).

\section{MATERIALS AND METHODS}

Design and simulation details: In this study, microstrip filter usingslotted patch resonators is designed using a dielectric constant of 10.8 and thickness of $1.27 \mathrm{~mm}$, conductor thickness of $35 \mu \mathrm{m}$ and loss tangent of 0.0023 . The topology of this resonator is illustrated in Fig. 2. The guided wave length ( $\lambda_{\mathrm{g} 0}$ is evaluated by Hong and Lancaster (1995a, b):

$$
\lambda_{\mathrm{g} 0}=\frac{\mathrm{c}}{\mathrm{f}_{0} \sqrt{\varepsilon_{\text {eff }}}}
$$

Where:

$\mathrm{c}=$ Light speed

$\mathrm{f}_{0}=$ Center frequency

$\varepsilon_{\text {eff }}$ is effective substrate value that can be determined from (Sun and Zhu, 2014; Yeo and Nwajana, 2013):

$$
\varepsilon_{\text {eff }}=\frac{\varepsilon_{\mathrm{r}}+1}{2}
$$

where, $\varepsilon_{\mathrm{r}}$ stands for relative substrate constant. The external dimension of slotted patch resonator is $9 \mathrm{~mm}$. The geometry of this resonator corresponds to active perturbation effect to the electromagnetic steadiness of the resonator configuration. Consequently, the electromagnetic distributions of the degenerated modes are going to be no longer orthogonal and combined to each other.

Based on this resonator, BPFs with cut, edge and without perturbation elements have been investigated using Microwave Office simulator as shown in Fig. 3. Circular corner perturbation patch has been used as 
(a)

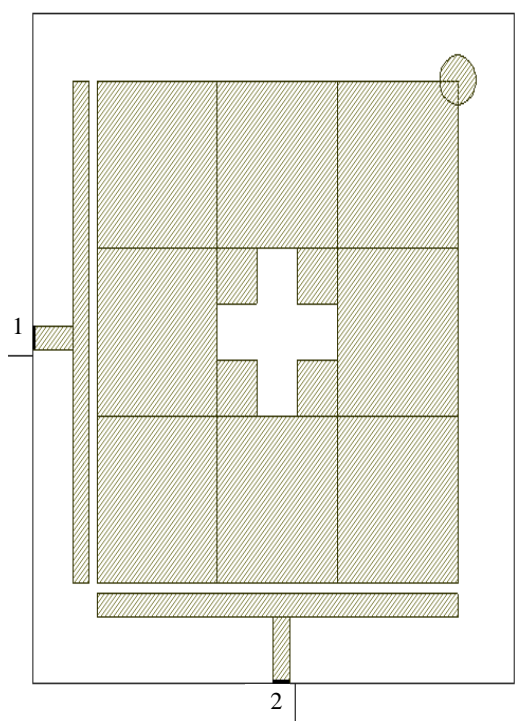

(b)

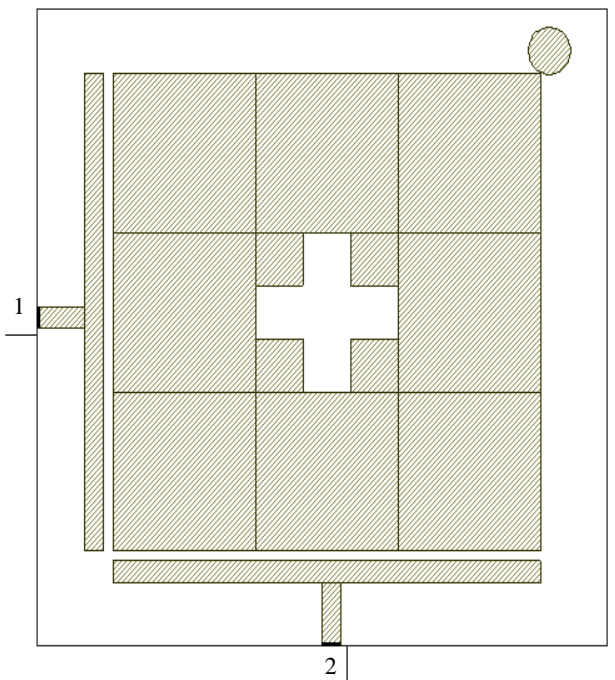

(c)

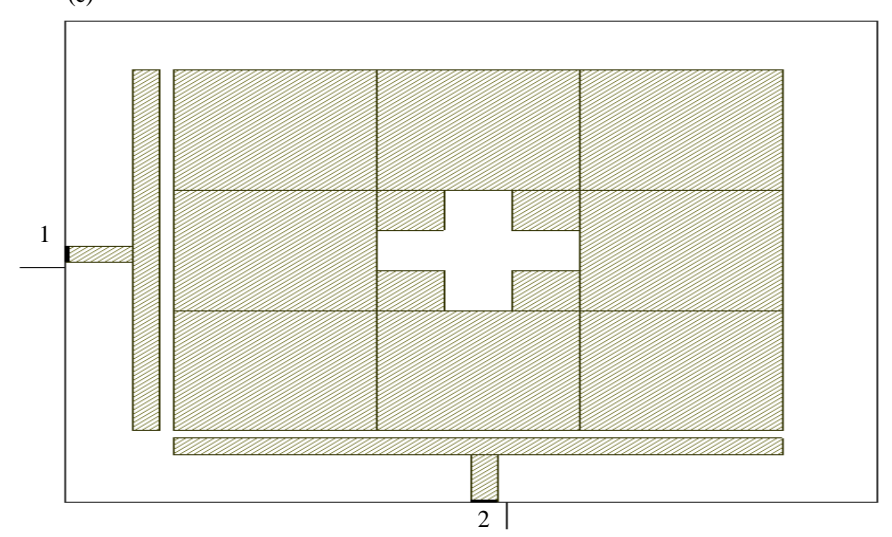

Fig. 3: Microstrip BPFs based on; a) Cut; b) Edge and c) Without perturbation elements

dual-mode induction element with a radius of $0.42 \mathrm{~mm}$ whereas the spacing between slotted patch resonator and input/output feeders is $0.2 \mathrm{~mm}$. The $\mathrm{I} / \mathrm{O}$ line length is $1 \mathrm{~mm}$. The full filter size is 12 by $12 \mathrm{~mm}^{2}(0.54$ $\lambda_{\mathrm{g}} \times 0.54 \lambda_{\mathrm{g}}$.

\section{RESULTS AND DISCUSSION}

The comparative simulation results of S21 and S1 1 parameters are shown in Fig. 4-5 and Table 1 in accordance with cut, edge and without perturbation elements.

From Fig. 4-5 and Table 1, the optimal frequency response that has finest return loss and insertion loss is found in a microstrip filter with cut perturbation element. The consequential S11 and S21 responses of the $\mathrm{BPF}$ is illustrated in Fig 6. This filter is designed at center frequency of $5.7 \mathrm{GHz}$ with return loss and insertion loss magnitudes of 1.7 and $11.5 \mathrm{~dB}$, respectively. Also, this designed filter has clearly narrow fractional bandwidth of $2.22 \%(127 \mathrm{MHz}$ from its center frequency).

It is detectable from this figure that there are dual transmission zeros that correspond to rejection band levels with magnitudes of 59 and $79 \mathrm{~dB}$ at 5.4 and $6.35 \mathrm{GHz}$ frequencies. Dual transmission poles at 5.67 and 5.735 $\mathrm{GHz}$ in $-3 \mathrm{~dB}$ passband region can be detected noticeably. On the subject of S12 curve, it comprises identical response of S21 curve, since, the filter design has self similarity.

Figure 7 presents the phase response of S21 and S1 1 parameters for the designed microstrip filter. It response has high quality of linearity that stands for very good characteristics in the filter design theory of quasi-elliptic 


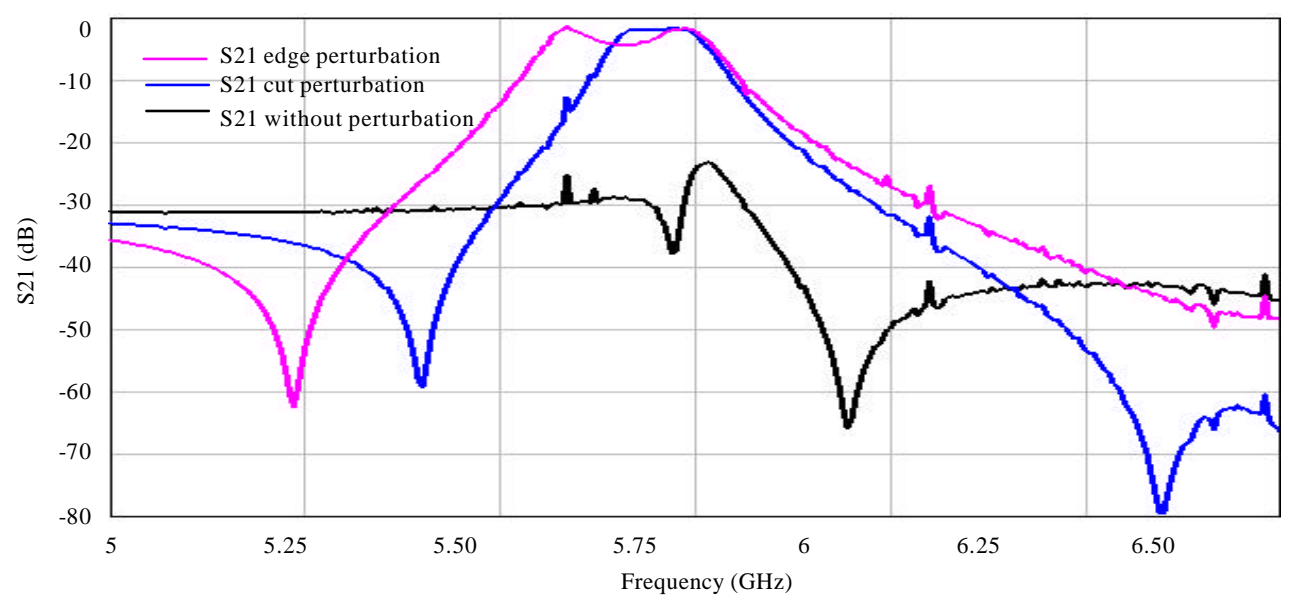

Fig. 4: S21 responses of the designed BPF based on $\mathrm{h}$ cut, edge and without perturbation elements

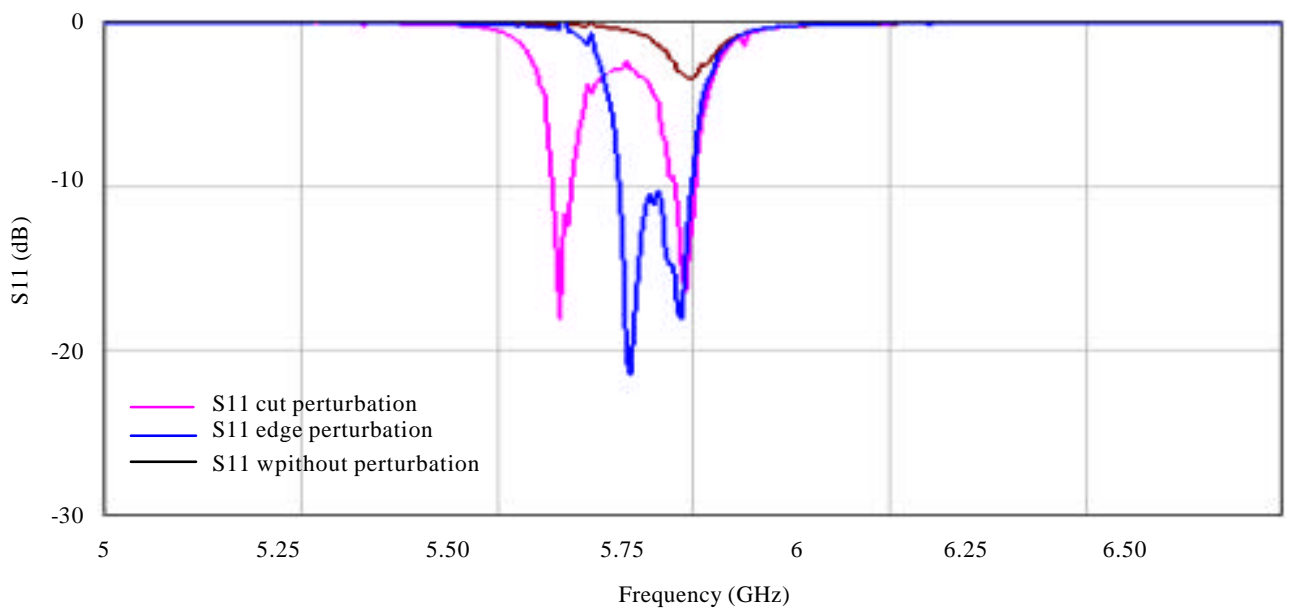

Fig. 5: S1 1 responses of the designed BPF based on cut, edge and without perturbation elements

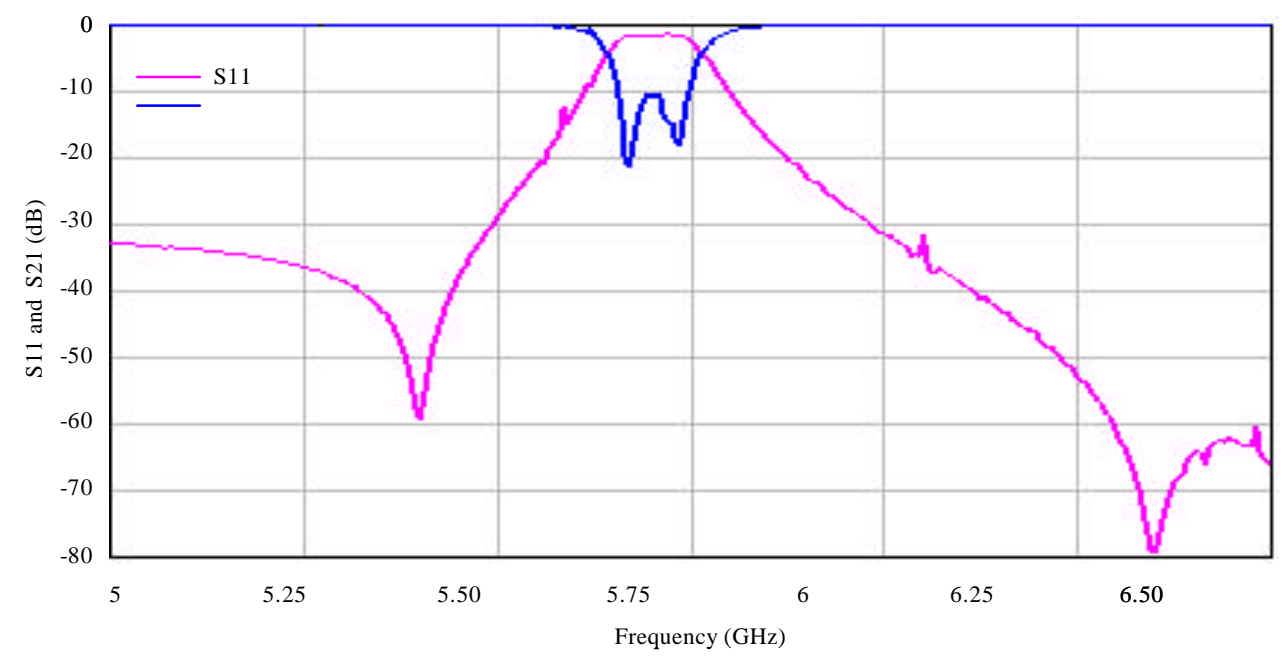

Fig. 6: The frequency responses for the projected microstrip filter 
Table 1: The electrical specifications of designed BPF based on cut, edge and without perturbation elements

\begin{tabular}{llll}
\hline Variables & Cut perturbation & Edge perturbation & Without perturbation \\
\hline Center frequency $(\mathrm{GHz})$ & 5.7 & 5.656 & 5.77 \\
Insertion loss $(\mathrm{dB})$ & 1.7 & 4.17 & 23 \\
Return loss $(\mathrm{dB})$ & 11.5 & 2.36 & 3.46 \\
Bandwidth range $(\mathrm{GHz})$ & $5.64-5.762$ & $5.555-5.769$ & - \\
\hline
\end{tabular}

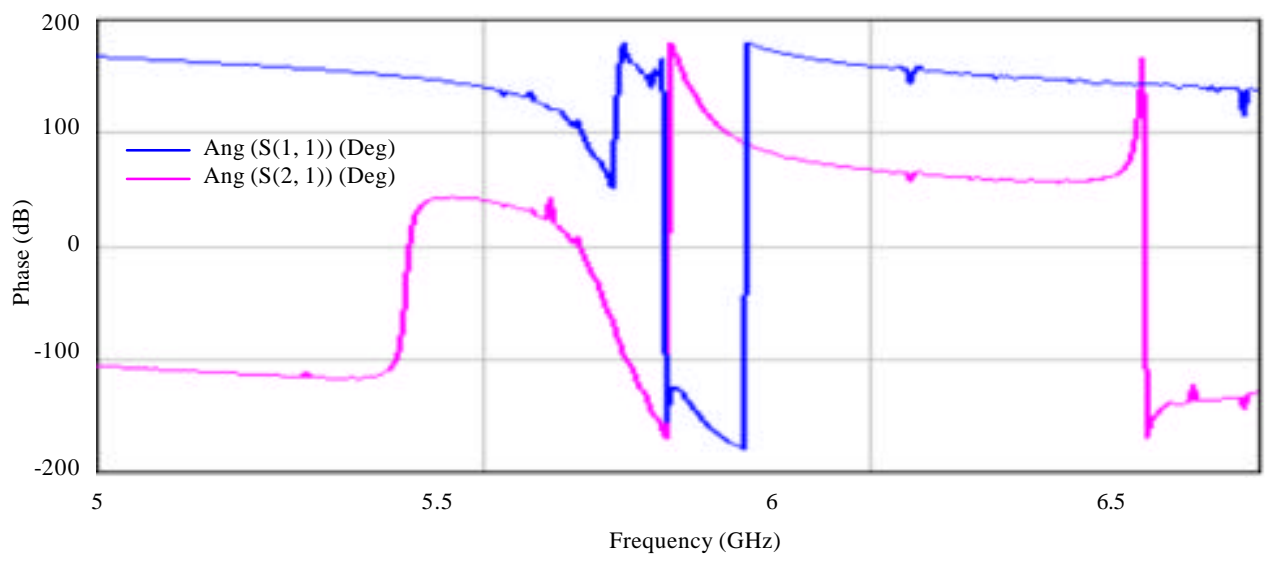

Fig. 7: The phase responses for the projected microstrip filter

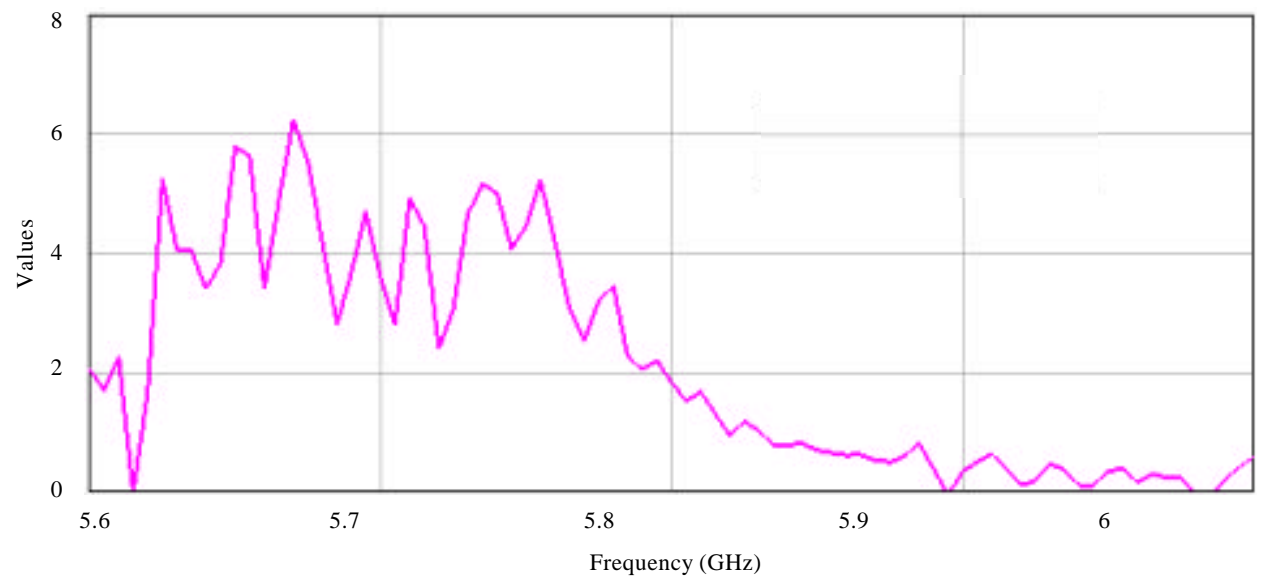

Fig. 8: The group delay of S21 parameter for the projected microstrip filter

response. Group delay within passband region is depicted in Fig. 8. It exhibits reasonable delay time during filtering operation with magnitudes of several nanoseconds.

\section{CONCLUSION}

A comprehensive survey on dual-mode microstrip $\mathrm{BPF}$ is presented to study the influence of reported dual-mode resonators with diverse kinds of perturbation elements on frequency response of microstrip filter. A new miniature microstrip BPF using new slotted dual-mode resonator has been simulated and analyzed. This filter has been designed at $5.7 \mathrm{GHz}$ with return loss and insertion loss values of 1.7 and $11.5 \mathrm{~dB}$. The surface area of the designed filter is 12 by $12 \mathrm{~mm}^{2}$. Furthermore, this designed filter has perceptibly narrow fractional bandwidth of $2.22 \%$. Consequently, the modeled microstrip filter can be realistic and integrated within numerous communication systems that require high quality factor filter with compact dimensions.

\section{REFERENCES}

Gupta, N., 2017. Effects of slots on microstrip patch antenna. Intl. Res. J. Eng. Technol., 4: 1132-1135.

Hong, J.S. and M.J. Lancaster, 1995b. Bandpass characteristics of new dual-mode microstrip square loop resonators. Electron. Lett., 31: 891-892. 
Hong, J.S. and M.J. Lancaster, 1995a. Microstrip bandpass filter using degenerate modes of a novel meander loop resonator. IEEE Microw. Guided Wave Lett., 5: 371-372.

Hong, J.S. and M.J. Lancaster, 2001. Microstrip Filters for RF/Microwave Application. 1st Edn., John Wiley and Sons Inc., New York.

Hong, J.S. and S. Li, 2004. Theory and experiment of dualmode microstrip triangular patch resonators and filters. IEEE. Trans. Microwave Theory Tech., 52: 1237-1243.

Kundu, A.C. and I. Awai, 2001. Control of attenuation pole frequency of a dual-mode microstrip ring resonator bandpass filter. IEEE. Trans. Microwave Theory Tech., 49: 1113-1117.

Mezaal, Y.S. and H.T. Eyyuboglu, 2016. Investigation of new microstrip bandpass filter based on patch resonator with geometrical fractal slot. PloS One, 11: $1-12$.

Mezaal, Y.S., H.T. Eyyuboglu and J.K. Ali, 2013. New dual band dual-mode microstrip patch bandpass filter designs based on Sierpinski fractal geometry. Proceedings of the 2013 3rd International Conference on Advanced Computing and Communication Technologies (ACCT), April 6-7, 2013, IEEE, Rohtak, India, ISBN:978-1-4673-5965-8, pp: 348-352.
Sheta, A.F., 2008. Narrow band compact non-degenerate dual-mode microstrip filter. Proceedings of the International Conference on Radio Science NRSC 2008 National, March 18-20, 2008, IEEE, Tanta, Egypt, ISBN:978-977-5031-95-2, pp: 1-7.

Sheta, A.F., N. Dib and A. Mohra, 2006. Investigation of new nondegenerate dual-mode microstrip patch filter. IEE. Proc.Microwaves Antennas Propag., 153: 89-95.

Singhal, P.K., S. Mathur and R.N. Baral, 2010. Compact narrow band non-degenerate dual-mode microstrip filter with etched square lattices. J. Electromagn. Anal. Appl., 2: 98-103.

Sun, S. and L. Zhu, 2014. Mode perturbations of a ring resonator for wideband and multiband filters. Proceedings of the 31th International Symposium on General Assembly and Scientific (URSI GASS) URSI, August 16-23, 2014, IEEE, Beijing, China, ISBN:978-14673-5225-3, pp: 1-4.

Wolff, I., 1972. Microstrip bandpass filter using degenerate modes of a microstrip ring resonator. Electron. Lett., 8: 302-303.

Yeo, K.S.K. and A.O. Nwajana, 2013. A novel microstrip dual-band bandpass filter using dual-mode square patch resonators. Prog. Electromagnet. Res., 36: 233247. 